\title{
The Major Histocompatibility Complex Class II-CD4 Immunologic Synapse in Alcoholic Hepatitis and Autoimmune Liver Pathology
}

\section{The Role of Aberrant Major Histocompatibility Complex Class II in Hepatocytes}

Jiajie G. Lu, Ping Ji, and Samuel W. French

From the Department of Pathology, Harbor-UCLA Medical Center, Torrance, California

Accepted for publication September 24, 2019.

Address correspondence to Jiajie G. Lu, M.D., Department of Pathology, Harbor-UCLA Medical Center, 1000 W Carson St., Torrance, CA 90502. E-mail: jlu3@dhs. lacounty.gov.

\begin{abstract}
The major histocompatibility complex class II (MHC II)-CD4 immunologic synapse is classically described between the T-cell receptor of CD4-positive lymphocytes and MHC II on antigen-presenting cells. This interaction and others between surrounding costimulatory and checkpoint molecules promote differentiation of naïve CD4 T lymphocytes into helper T cells subtypes, including types 1, 2, and 17 helper T cells, that have more tailored immunologic responses. Although MHC II is mainly produced by professional antigen-presenting cells, it can be aberrantly produced by other cell types, including hepatocytes in various liver pathologies, such as autoimmune hepatitis and alcoholic hepatitis. This can lead to direct targeting of hepatocytes by CD4-positive lymphocytes, which form an immunologic synapse with the hepatocyte. The lymphocytes internalize the MHC II-CD4 complexes in a phagocytosis-like mechanism and in the process eat the hepatocyte piece by piece. We review the evidence for this mechanism and the role of these autoimmune responses in various liver diseases, including alcoholic hepatitis, autoimmune hepatitis, and primary biliary cirrhosis. The role of aberrant MHC II in malignancy, including hepatocellular carcinoma, is also reviewed. Further understanding of this mechanism can lead to better understanding of the immune mechanisms involved in these liver pathologies, with potential diagnostic and therapeutic applications. (Am J Pathol 2020, 190: 25-32; https://doi.org/10.1016/j.ajpath.2019.09.019)
\end{abstract}

\section{Historical Context and Current Understanding of the Immunologic Synapse}

Initially, it was recognized that the major histocompatibility complex (MHC) is required for specific recognition of antigen by T cells. ${ }^{1}$ Solving the crystal structure of major histocompatibility complex shed further light on this mechanism as it demonstrated how a foreign peptide antigen fit into the MHC complex. ${ }^{2}$ Other molecules required for T-cell activation were also discovered; however, it was unclear how these various signals could cause T-cell activation as activating them alone was not sufficient for T-cell activation.
The first description of the immunologic synapse in 1995 furthered our understanding in $1995 .{ }^{4}$ Using digital imaging to analyze antigen-specific interactions between individual $\mathrm{T}$ cells and antigen-presenting cells, the authors showed that rather than even distribution of key molecules involved in T-cell activation, there were segregated, concentrated threedimensional clusters of molecules. Subsequent studies further characterized the immunologic synapse. The classic

Supported by NIH/National Institute on Alcohol Abuse and Alcoholism grant UO-21898-05 (S.W.F.).

Disclosures: None declared. 
description is a bull's eye with a central major histocompatibility (MHC)-T-cell receptor structure surrounded by a ring of lymphocyte function-associated antigen- 1 and intercellular adhesion molecule 1 adhesion molecules. ${ }^{5}$ Further outside is a ring that includes CD45. ${ }^{6}$ In T cells, there are two main types of immunologic synapses, depending on the type of MHC complex and T-cell coreceptor: MHC II-CD4 and MHC $\mathrm{I}-\mathrm{CD} 8$. CD8 T cells have cytotoxic function toward MHC I loaded with proteasome-degraded peptide. Our focus is on the MHC II-CD4 immunologic synapse.

The MHC II-CD4 immunologic synapse results in helper T-cell activation due to presentation of endocytosed antigen on the MHC II complex. ${ }^{7}$ Specialized antigenpresenting cells, such as dendritic cells and macrophages, endocytose antigens and load them onto MHC II, where they are recognized by naïve CD4-positive $\mathrm{T}$ lymphocytes. First, adhesion molecules pull the $\mathrm{T}$ cell and the target cell together. ${ }^{8}$ Then, the T-cell receptor binds to the MHC II-peptide complex, with CD4 functioning as a coreceptor. The MHC II-CD4 immunologic synapse is $15 \mathrm{~nm}$ across. ${ }^{9}$ Positive costimulatory molecules CD40, CD40 ligand, CD80/86, and CD28 aid the interaction, ${ }^{10}$ whereas negative costimulators programmed death ligand 1 (PDL1), program cell death protein 1 (PD-1), CD80, and CTLA-4 attenuate the interaction. ${ }^{11,12}$ Successful activation of CD4 T cells leads to differentiation into helper $\mathrm{T}$ cell subtypes, including types 1, 2, and 17 helper $\mathrm{T}$ cells (Th1, Th2, and Th17, respectively), that have more tailored immune responses.

\section{Aberrant MHC II Production in Hepatocytes and Other Nonantigen-Presenting Cells}

Although MHC II is classically thought to be found on antigen-presenting cells, it can also be present on other cell types. In skin, breast, lung, and kidney tissues, MHC II is normally present. ${ }^{13}$ MHC II can also be aberrantly present in various liver pathologies. In viral hepatitis and autoimmune hepatitis, hepatocytes aberrantly have higher levels of MHC II. ${ }^{14,15}$ In a series of pediatric liver biopsies, MHC II was aberrantly present on hepatocytes in 3 of 10 cases of autoimmune hepatitis and 5 of 9 cases of primary sclerosing cholangitis, but not in biliary atresia, $\alpha$ 1 antitrypsin deficiency, idiopathic neonatal hepatitis, or normal liver. ${ }^{16}$ Interestingly, the cases that showed aberrant hepatocyte MHC II were untreated patients. Also, hepatocytes in human biopsies of alcoholic hepatitis have increased levels of MHC II but not those in nonalcoholic steatohepatitis $^{17,18}$ (Figure 1). In our study, the biopsies of alcoholic hepatitis also showed higher levels of interferon$\gamma$ and IL- $1 \alpha$ compared with those of nonalcoholic steatohepatitis and normal controls.

The aberrant production of MHC II is triggered by interferon- $\gamma .{ }^{15}$ Once hepatocytes produce MHC II, they can express costimulatory molecules and activate CD4-positive lymphocytes, toward either a Th1 or a Th2 phenotype. ${ }^{19}$ By itself, aberrant MHC II production is not sufficient to cause autoimmune hepatitis, ${ }^{19}$ but it may enhance an already existing autoimmune response. It may also help bypass the normally immunotolerant microenvironment of the liver. ${ }^{20}$ Interestingly, MHC II production is also present on bile duct epithelium in primary biliary cirrhosis and primary sclerosing cholangitis. ${ }^{21}$

Aberrant MHC II production is also seen in other organs, usually in the autoimmune setting, with the level of expression tending to correlate with disease severity. In autoimmune thyroiditis, aberrant MHC II production is seen in thyrocytes and is associated with a slight increase in the severity of the autoimmune response compared with cases with no MHC II expression in thyrocytes. ${ }^{22}$ Similar to autoimmune hepatitis, MHC II production by itself was not
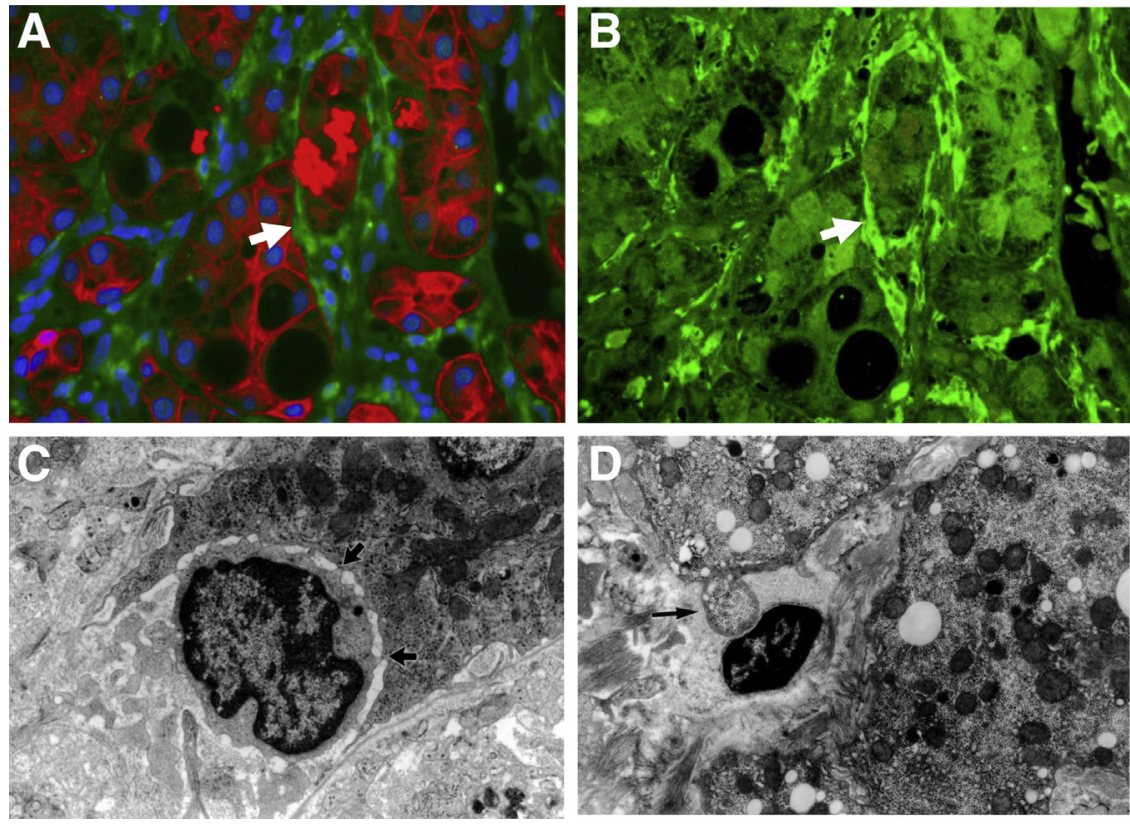

Figure 1 A: Aberrant major histocompatibility complex class II (MHC II) production (green) in a liver biopsy with alcoholic hepatitis. Note the Mallory body (arrow), which stains positively for CAM5.2 (red) in the hepatocyte coated with MHC II. Nuclei are stained with DAPI (blue). B: Same biopsy with only MHC II shown (green); note the pericellular distribution of MHC II in the hepatocyte with Mallory body (arrow). C: Electron micrograph of a lymphocyte indenting a hepatocyte and forming multiple immunologic synapses (arrows) with the hepatocyte. D: Electron micrograph from a case of autoimmune hepatitis with a lymphocyte surrounding a bleb (arrow) from a hepatocyte. C and D: Adapted from Wang et $\mathrm{al}^{18}$ with permission from Experimental and Molecular Pathology. Original magnification: $\times 780$ (A and B); $\times 700$ (C); $\times 24,000$ (D). 
sufficient to trigger autoimmune thyroiditis. Aberrant MHC II production is also seen in diabetic insulitis, ${ }^{23}$ autoimmune uveitis, ${ }^{24}$ adrenal atrophy, ${ }^{25}$ autoimmune gastritis, ${ }^{26}$ and rheumatoid arthritis-like joint disease. ${ }^{27}$ Misfolded cellular proteins are transported to the cell surface by MHC II molecules, a possible mechanism for autoimmune response. ${ }^{28}$ In the case of rheumatoid arthritis-like joint disease, transgenic mice that aberrantly produce MHC II did not spontaneously develop autoimmune joint disease, but they were highly susceptible to immunization with low doses of foreign protein (bovine collagen), whereas negative control mice did not respond to this stimulus. The aberrantly MHC II-producing mice developed joint disease phenotypically and histologically akin to rheumatoid arthritis. ${ }^{27}$

Aberrant MHC II production can also be seen in the neoplastic setting. Melanoma cells often aberrantly produce MHC II, which leads to a dampening of CD8 T-cell antitumor activity. ${ }^{29}$ In papillary thyroid carcinoma, higher levels of MHC II are inversely correlated with recurrence of carcinoma. ${ }^{30}$ In a study of triple-negative breast cancer, the presence of MHC II components is associated with progressionfree survival. ${ }^{31}$ Among the genes with significantly higher expression in no relapse patients were CIITA, the activator of MHC II; CD74, the chaperone for MHC II; and various human leukocyte antigen $(H L A)$ genes that express the components of MHC II. Histologically, this was correlated with tumor cells staining for CD74 and HLA-DPB1 protein and lymphocytic infiltrate. In addition to the aforementioned examples, MHC II production can be seen in tumor cells in colorectal carcinoma, ${ }^{32}$ squamous cell carcinoma, ${ }^{33}$ and osteosarcoma. ${ }^{34}$

\section{The MHC II-CD4 Immunologic Synapse and Piecemeal Necrosis}

To further understand the role of aberrant MHC II in liver pathology, it is necessary to understand piecemeal necrosis, which is the historical term for interface hepatitis. ${ }^{35}$ Normally, there is a sharp demarcation between the portal tract and surrounding cords of hepatocytes, which is termed the limiting plate. In interface hepatitis, this demarcation is disrupted and a predominantly mononuclear inflammatory cell infiltrate involves the hepatic sinusoids in the periportal regions. ${ }^{36}$ The hepatocytes in this area are gradually destroyed. This phenomenon is seen in various conditions, including autoimmune hepatitis, viral hepatitis, primary biliary cirrhosis, and liver transplant rejection. ${ }^{37}$ It is also observed in alcoholic hepatitis. ${ }^{38}$

The mechanism of hepatocyte damage varies by disease. However, there is a mixture of CD4- and CD8-positive T-lymphocyte infiltrate, of which CD4 lymphocytes are usually predominant. ${ }^{36} \mathrm{~B}$ cells are uncommon in these lymphocytic infiltrates. ${ }^{39}$ More important, when CD4-positive lymphocytes are activated through the MHC II-CD4 immunologic synapse, they can absorb molecules from the antigen-presenting cell, including $\mathrm{MHC}$ and the costimulatory molecule B7. ${ }^{40}$ This process is dependent on the actin cytoskeleton. ${ }^{41}$ It is also dependent on TC21 and RhoG, a GTPase involved in phagocytosis. ${ }^{42}$ Normally, CD4 $\mathrm{T}$ lymphocytes interact with antigen-presenting cells, but if hepatocytes can aberrantly express MHC II, this raises the question of whether CD4 lymphocytes can directly attack and phagocytose hepatocytes. Indeed, with their T-cell receptors, $\mathrm{T}$ cells can phagocytose beads 1 to $6 \mu \mathrm{m}$ in diameter. ${ }^{42}$ For comparison, a hepatocyte is 20 to $30 \mu \mathrm{m}$ in width; thus, the size and scale of this mechanism seem plausible.

To further investigate, we previously performed electron microscopy studies for piecemeal necrosis in viral hepatitis, autoimmune hepatitis, primary biliary cirrhosis, and steatohepatitis. ${ }^{18,43}$ Piecemeal necrosis requires formation of an immunologic synapse between the lymphocyte and hepatocyte as they closely associate with each other. The hepatocyte indents at the interface with the lymphocyte, and the lymphocytes eat hepatocytes piece by piece and digest them in their lysosomes. Microscopically one sees loss of liver cell volume and irregular pieces (nubbins) of hepatocytes dispersed through the sinusoidal area devoid of nuclear material. By electron microscopy, one sees lymphocytes indenting the cytoplasm and approaching the nucleus of hepatocytes. Other images show attachments between the plasma membranes of lymphocyte and hepatocyte, strongly suggestive of immunologic synapse formation. Furthermore, lymphocytes are seen surrounding blebs of neighboring hepatocytes, as though in the process of phagocytosing them, and phagosomes are seen within the cytoplasm of activated lymphocyte. This phenomenon was named troxis necrosis, deriving from the Greek verb trogo, meaning to eat, chew, gnaw, to reflect direct phagocytosis of hepatocytes by lymphocytes. ${ }^{18}$

MHC II is aberrantly produced in various hepatitis pathologies, and CD4 lymphocytes are the predominant lymphocytic infiltrate; the process of piecemeal necrosis is likely due to CD4 lymphocytes directly attacking and eating hepatocytes. For instance, CD4-positive lymphocytes closely associate with hepatocytes in alcoholic hepatitis, and electron microscopy showed them forming immunologic synapses with hepatocytes. ${ }^{17}$

Interestingly, a subset of CD4 lymphocytes has been shown to have direct cytotoxic activity. ${ }^{44,45}$ The CD4-positive cytotoxic $\mathrm{T}$ cells can derive from any subset of T-helper cells but usually arise from Th1 cells, which secrete interferon- $\gamma$ and promote a cell-mediated response. ${ }^{46}$ The presence of CD4 cytotoxic lymphocytes may be protective in nature as some viruses can cause their host cells to underexpress MHC class I, thus evading CD8 cytotoxic T-lymphocyte response. ${ }^{47}$

\section{Immunologic Synapse Formation in Autoimmune Hepatitis}

Autoimmune hepatitis is diagnosed by a combination of clinical and pathologic features. The typical biopsy findings 
include a heavy portal inflammatory infiltrate and interface hepatitis, with plasma cells and eosinophils accompanying lymphocytes. ${ }^{48}$ Lobular hepatitis may also be seen. Druginduced hepatitis has varying appearance, depending on the medication, but can show similar histologic features to autoimmune hepatitis.

The inflammatory infiltrate of autoimmune hepatitis typically shows CD4-positive lymphocytes in the portal tracts. The lymphocytic infiltrate at the area of interface hepatitis varies in immunophenotype. In one study, CD8-positive lymphocytes were predominant in the areas of interface hepatitis in contrast to the CD4-rich portal tracts. ${ }^{20}$ In another study, an analysis of $189 \mathrm{~T}$-cell clones from four autoimmune hepatitis liver biopsies showed predominantly $\mathrm{CD}^{+} / \mathrm{CD}^{-}$ clones, with a CD4/CD8 ratio of 2.0 to $3.8 .^{48}$ Interestingly, some of the CD4-positive T-cell clones showed cytolytic activity, lending further support to the concept of cytotoxic $\mathrm{T}$ cells. ${ }^{49}$ An additional study of 12 pediatric liver biopsies for patients with autoimmune hepatitis also showed predominantly CD4-positive lymphocytes in the portal tract compared with CD8-positive lymphocytes. ${ }^{50}$ Interestingly, the autoimmune reaction occurs in spite of increased checkpoint inhibitor PD-1 expression on Kupffer cells. ${ }^{51}$

Immunologic synapse formation has been shown in autoimmune hepatitis with CD4-positive lymphocytes. ${ }^{43}$ In addition, in a case of drug-induced hepatitis secondary to immunomodulatory medications, CD4 lymphocytes were seen forming immunologic synapses with hepatocytes. ${ }^{52}$ This phenomenon was also seen in cases of hepatotoxicity due to black cohosh ${ }^{53}$ and from taking Herbalife weight loss supplements. ${ }^{54}$ Immunologic synapse formation from CD4-positive lymphocytes and MHC II-producing hepatocytes may be an important mechanism in autoimmune hepatitis that leads to direct phagocytosis of hepatocytes.

\section{Autoimmune Mechanisms in Alcoholic Hepatitis}

Alcoholic hepatitis is characterized histologically by steatosis, Mallory body formation, pericellular and perivenular fibrosis, and hepatocyte ballooning. ${ }^{48}$ In addition, it is associated with an immune response that is multifactorial in nature. Previously characterized mechanisms include lipopolysaccharide-induced acute inflammation due to increased gut epithelial membrane permeability, ${ }^{55}$ direct response to free fatty acids, ${ }^{56}$ and reactive oxygen species formation. ${ }^{57}$ In addition, the response is partly antibody mediated. Autoantibodies can be formed against cytochrome proteins CYP2E1 and CYPEA4 $4{ }^{58,59}$ hydroxyethyl radical protein adducts, ${ }^{60}$ and acetaldehyde binding to cellular proteins. $^{61}$

Overall, these mechanisms trigger a primarily acute inflammatory response with neutrophils and macrophages. Lymphocyte response in alcoholic hepatitis is less well described. ${ }^{62}$ However, increased levels of MHC II have recently been shown on hepatocellular membranes in biopsies of alcoholic hepatitis (Figure 1). Immunologic synapse formation has also been observed between CD4-positive lymphocytes and hepatocytes in these biopsies. Combined with previous findings regarding piecemeal necrosis in steatohepatitis, direct eating of hepatocytes by CD4-positive lymphocytes may be an important autoimmune mechanism seen in alcoholic hepatitis. ${ }^{18}$

A proposed model for this autoimmunity can be seen in Figure 2. Exposure to alcohol leads to development of autoantibodies, which activate Th1 helper $\mathrm{T}$ cells in the traditional antigen-presenting cell pathway. Th1 cells secrete interferon- $\gamma$, leading to increased levels of MHC II in hepatocytes, which allows direct targeting of hepatocytes by CD4-positive cells. As hepatocytes are eaten and degraded, inflammatory cytokines, such as IL- $1 \alpha$, are released, which further activate CD4 lymphocytes, leading to a positive feedback loop. In addition, IL- $1 \alpha$ recruits macrophages, which themselves secrete interferon- $\gamma$, leading to further MHC II expression on hepatocytes and additional positive feedback.

\section{Autoimmune Mechanisms in Primary Biliary Cirrhosis}

Primary biliary cirrhosis is characterized pathologically by initial heavy portal inflammation with bile duct damage, followed by ductular proliferation, scarring with bridging fibrosis, and cirrhosis. ${ }^{54}$ Granulomas are sometimes seen. The inflammatory infiltrate is predominantly lymphocytic, with mostly CD4-positive $\mathrm{T}$ cells, and the overall appearance may mimic other forms of chronic hepatitis.

One study of 15 liver biopsies from patients with primary biliary cirrhosis showed expression of MHC II subregion genes (HLA-DP, HLA-DR, and $H L A-D Q$ ) on bile duct epithelial cells in early-stage disease. ${ }^{63}$ In advanced-stage disease, this expression decreased. In addition, four biopsies showed hepatocytes staining for HLA-DR. Another study found increased levels of MHC class I molecules in addition to aberrant HLA-DR expression. ${ }^{64}$ Furthermore, certain alleles of HLA-DR and HLA-DQ are associated with increased risk for primary biliary cirrhosis, whereas other alleles are considered protective. ${ }^{65}$ Thus, it appears that aberrant MHC II on bile duct epithelium plays a role in the early-stage pathology of this disease.

\section{Summary and Diagnostic and Clinical Applications}

Aberrant MHC II on hepatocytes or bile duct epithelium is a mechanism to amplify autoimmune response by CD4-positive lymphocytes and even lead to direct targeting and destruction by cytotoxic CD4 T lymphocytes. The lymphocytes form MHC II-CD4 immunologic synapses with hepatocytes and, in the process of 


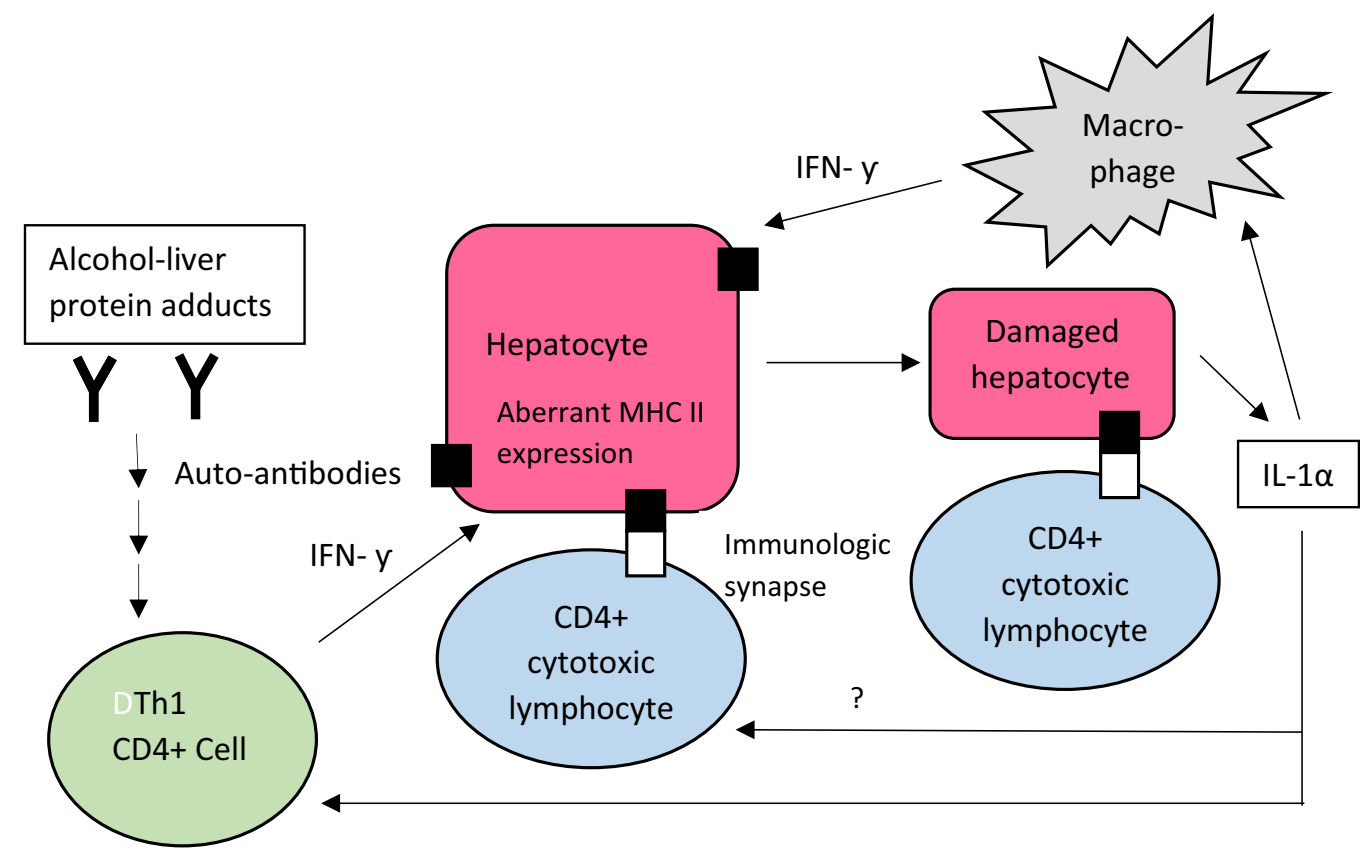

Figure 2 Model for autoimmune mechanisms in alcoholic hepatitis. Autoantibodies to alcohol-liver protein adducts lead to a type 1 helper T-cell (Th1) response, leading to secretion of interferon- $\gamma$ (IFN- $\gamma$ ). IFN- $\gamma$ causes aberrant major histocompatibility complex class II (MHC II) production on hepatocytes, leading to targeting by CD4-positive cytotoxic lymphocytes. As hepatocytes are destroyed, they release IL-1 $\alpha$, which, among other effects, further enhances antigen-activated $\mathrm{CD}_{4}^{+}$T-cell response, generating a positive feedback loop.

internalizing these complexes, phagocytose the hepatocytes piece by piece. This is an additional autoimmune mechanism that appears to be involved in various pathologies, including autoimmune hepatitis, alcoholic hepatitis, and primary biliary cirrhosis.

There are several follow-up investigations. One is whether MHC II immunohistochemistry can be used for diagnostic purposes. As there is considerable overlap in the histologic appearance of viral hepatitis, autoimmune hepatitis, primary biliary cirrhosis, and primary sclerosing cholangitis, the presence of aberrant MHC II could be used to help differentiate between these conditions. In particular, viral hepatitis and autoimmune hepatitis would show aberrant MHC II on hepatocytes, whereas primary biliary cirrhosis and primary sclerosing cholangitis would show MHC II on bile duct epithelium. Given data from previous studies, this marker would likely have lower sensitivity but high specificity; it may have higher sensitivity in untreated patients. ${ }^{16}$

Second, we can investigate whether MHC II is aberrantly produced in hepatocellular carcinoma. One study investigated whether differential expression of individual genes and signaling pathway components correlated with recurrencefree survival for hepatocellular carcinoma. ${ }^{66}$ The authors found that MHC II-related antigen-presenting pathways significantly correlated with longer recurrence-free survival time. They note that MHC II molecules were found on CD68-positive (macrophage) and CD45-positive (lymphocyte) cells. Whether MHC II is present on tumor cells remains to be investigated, in addition to whether lymphocytes can be seen on electron microscopy, directly forming immunologic synapses with tumor cells.

Recent clinical trials have investigated use of pembrolizumab in advanced hepatocellular carcinoma. Pembrolizumab is an inhibitor of PD-1, a receptor that is expressed in lymphocytes and attenuates T-cell activation via the immunologic synapse. A nonrandomized, openlabel, phase 2 trial of pembrolizumab for patients with hepatocellular carcinoma previously treated with sorafenib found that $17 \%$ of 104 treated patients had partial or complete response to the medication. ${ }^{67}$ This finding prompted a fast-track approval of pembrolizumab for this indication by the Food and Drug Administration. The phase 3 trial of pembrolizumab showed a trend toward improved overall survival and progression-free survival, although these findings were not statistically significant. ${ }^{68}$ Why do only some patients respond to pembrolizumab? One hypothesis is that some patients had higher levels of tumor MHC II; even if the inhibitory signal from PD-L1 is diminished by the medication, lymphocytes may not adequately target tumor cells if there is insufficient tumor MHC II. As pembrolizumab is an expensive medication, stratification of potential therapeutic response by a marker such as MHC II may lead to more cost-effective management.

Finally, in the setting of autoimmune liver pathologies, one wonders what mechanisms can attenuate MHC II-CD4 immunologic synapse formation and piecemeal necrosis. The adverse effects of CTLA-4 and PD- 1 checkpoint inhibitors include reactions resembling autoimmune disease, including pruritis, rash, colitis, inflammation pneumonitis, 
and interstitial nephritis. ${ }^{69}$ These medications can also worsen already existing autoimmune disease, ${ }^{70}$ such as psoriasis. $^{71}$ Type 1 diabetes has even been induced in the context of this medication. $^{72}$ It stands to reason that the opposite action, enhancing checkpoint inhibitor CTLA-4 and PD-1 activity, would have the opposite effect of reducing autoimmunity. In addition, one can consider blocking the costimulatory molecule activity of CD28 and CD80, which theoretically would have a similar effect as enhancing checkpoint inhibitor activity. Further studies are needed to evaluate the feasibility and efficacy of such targeted therapies.

\section{References}

1. Blanden RV, Doherty PC, Dunlop MB, Gardner ID, Zinkernagel RM, David CS: Genes required for cytotoxicity against virus-infected target cells in K and D regions of H-2 complex. Nature 1975, 254: 269-270

2. Bjorkman PJ, Saper MA, Samraoui B, Bennett WS, Strominger JL, Wiley DC: Structure of the human class I histocompatibility antigen, HLA-A2. Nature 1987, 329:506-512

3. Monks CR, Kupfer H, Tamir I, Barlow A, Kupfer A: Selective modulation of protein kinase C-theta during T-cell activation. Nature 1997, 385:83-86

4. Monks CR, Freiberg BA, Kupfer H, Sciaky N, Kupfer A: Threedimensional segregation of supramolecular activation clusters in $\mathrm{T}$ cells. Nature 1998, 396:82-86

5. Dustin ML, Springer TA: Lymphocyte function-associated antigen-1 (LFA-1) interaction with intercellular adhesion molecule-1 (ICAM-1) is one of at least three mechanisms for lymphocyte adhesion to cultured endothelial cells. J Cell Biol 1988, 107:321-331

6. Johnson KG, Bromley SK, Dustin ML, Thomas ML: A supramolecular basis for CD45 tyrosine phosphatase regulation in sustained T cell activation. Proc Natl Acad Sci U S A 2000, 97:10138-10143

7. Dustin ML: The immunological synapse. Cancer Immunol Res 2015, 11:1023-1033

8. Springer TA: Adhesion receptors of the immune system. Nature 1990, 346:425-434

9. Garcia KC, Degano M, Stanfield RL, Brunmark A, Jackson MR, Peterson PA, Teyton L, Wilson IA: An alphabeta T cell receptor structure at $2.5 \mathrm{~A}$ and its orientation in the TCR-MHC complex. Science 1996, 274:209-219

10. Bromley SK, Iaboni A, Davis SJ, Whitty A, Green JM, Shaw AS, Weiss A, Dustin ML: The immunological synapse and CD28-CD80 interactions. Nat Immunol 2001, 2:1159-1166

11. Krummel MF, Allison JP: CTLA-4 engagement inhibits IL-2 accumulation and cell cycle progression upon activation of resting T cells. J Exp Med 1996, 183:2533-2540

12. Ishida $\mathrm{Y}$, Agata $\mathrm{Y}$, Shibahara K, Honjo T: Induced expression of PD1, a novel member of the immunoglobulin gene superfamily, upon programmed cell death. EMBO J 1992, 11:3887-3895

13. Drozina G, Kohoutek J, Jabrane-Ferrat N, Peterlin BM: Expression of MHC II genes. Curr Top Microbiol Immunol 2005, 290:147-170

14. Dienes HP, Hutteroth T, Hess G, Meuer SC: Immunoelectron microscopic observations on the inflammatory infiltrates and HLA antigens in hepatitis B and non-A, non-B. Hepatology 1987, 7: $1317-1325$

15. Franco A, Barnaba V, Natali P, Balsano C, Musca A, Balsano F: Expression of class I and class II major histocompatibility complex antigens on human hepatocytes. Hepatology 1988, 8:449-454

16. Lobo-Yeo A, Senaldi G, Portmann B, Mowat AP, Mieli-Vergani G, Vergani D: Class I and class II major histocompatibility antigen expression on hepatocytes: a study in children with liver disease. Hepatology 1990, 12:224-232

17. Lu JG, Iyasu A, French B, Tillman B, French SW: Overexpression of MHCII by hepatocytes in alcoholic hepatitis (AH) compared to nonalcoholic steatohepatitis (NASH) and normal controls. Alcohol 2019, [Epub ahead of print] doi:10.1016/j.alcohol.2019.08.008

18. Wang MY, Morgan T, Lungo W, Wang L, Sze GZ, French SW: "Piecemeal" necrosis: renamed troxis necrosis. Exp Mol Pathol 2001, $71: 137-146$

19. Herkel J, Jagemann B, Wiegard C, Garcia Lazaro JF, Lueth S, Kanzler S, Blessing M, Schmitt E, Lohse AW: MHC class-Il expressing hepatocytes function as antigen-presenting cells and activate specific CD4 T lymphocytes. Hepatology 2003, 37:1079-1085

20. Horst AK, Neumann K, Diehl L, Tiegs G: Modulation of liver tolerance by conventional and nonconventional antigen-presenting cells and regulatory immune cells. Cell Mol Immunol 2016, 13:277-292

21. Hashimoto E, Lindor KD, Homburger HA, Dickson ER, Czaja AJ, Wiesner RH, Ludwig J: Immunohistochemical characterization of hepatic lymphocytes in primary biliary cirrhosis in comparison with primary sclerosing cholangitis and autoimmune chronic active hepatitis. Mayo Clin Proc 1993, 68:1049-1055

22. Kimura H, Kimura M, Tzou SC, Chen YC, Suzuki K, Rose NR, Caturegli P: Expression of class II major histocompatability complex molecules on thyrocytes does not cause spontaneous thyroiditis but mildly increases its severity after immunization. Endocrinology 2005, 146:1154-1162

23. Londei M, Lamb JR, Bottazzo GF, Feldmann M: Epithelial cells expressing aberrant MHC class II determinants can present antigen to cloned human T cells. Nature 1984, 312:639-641

24. Lipski DA, Dewispelaere R, Foucart V, Caspers LE, Defrance M, Bruyns C, WIllermain F: MHC class II expression and potential antigen-presenting cells in the retinal during experimental autoimmune uveitis. J Neuroinflammation 2017, 14:136

25. Jackson R, McNicol AM, Farquharson M, Foulis AK: Class II MHC expression in normal adrenal cortex and cortical cells in autoimmune Addison's disease. J Pathol 1988, 155:113-120

26. Bockerstett KA, Wong CF, Koehm S, Ford EL, DiPaolo RJ: Molecular characterization of gastric epithelial cells using flow cytometry. Int J Mol Sci 2018, 19:1096

27. Kanazawa S, Ota S, Sekine C, Tada T, Otsuka T, Okamoto T, Sonderstrup G, Peterlin BM: Aberrant MHC class II expression in mouse joints leads to arthritis with extraarticular manifestations similar to rheumatoid arthritis. Proc Natl Acad Sci U S A 2006, 103 : $14465-14470$

28. Arase H, Jin H, Tanimura K, Suenaga T, Arase N, Lanier LL: Cellular misfolded proteins transported to the cell surface by aberrantly expressed MHC class II molecules are major autoantibody targets in autoimmune diseases. J Immunol 2017, 198 Suppl 1. 156.20

29. Donia M, Andersen R, Kjeldsen JW, Fagone P, Munir S, NIcoletti F, Andersen MH, Thor Straten P, Svane IM: Aberrant expression of MHC class II in melanoma attracts inflammatory tumor-specific $\mathrm{CD} 4+\mathrm{T}$-cells, which dampen $\mathrm{CD} 8+\mathrm{T}$ cell antitumor reactivity Cancer Res 2015, 75:3747-3759

30. Jo YS, Lee JC, Li S, Choi YS: Significance of the expression of major histocompatibility complex class II antigen, HLA-DR and -DQ, with recurrence of papillary thyroid cancer. Int $\mathbf{J}$ Cancer 2008, 122: 785-790

31. Forero A, Li Y, Chen D, Grizzle WE, Updike KL, Merz ND, DownsKelly E, Burwell TC, Vaklavas C, Buchsbaum DJ, Myers RM, LoBuglio AF, Varley KE: Expression of the MHC class II pathway in triple-negative breast cancer tumor cells is associated with a good prognosis and infiltrating lymphocytes. Cancer Immunol Res 2016, 4 : 390-399

32. Degener T, Momburg F, Moller P: Differential expression of HLADR, HLA-DP, HLA-DQ and associated invariant chain (Ii) in normal colorectal mucosa, adenoma and carcinoma. Virchows Arch A Pathol Anat Histopathol 1988, 412:315-322 
33. Nasman A, Andersson E, Marklund L, Tertipis N, HammarstedtNordenvall L, Attner P, Nyberg T, Masucci GV, Munck-Wikland E, Ramqvist T, Dalianis T: HLA class I and II expression in oropharyngeal squamous cell carcinoma in relation to tumor HPV status and clinical outcome. PLoS One 2013, 8:e77025

34. Zhou Q, Xu J, Zhao J, Zhang S, Pan W: Downregulation of CD99 and upregulation of human leukocyte antigen class II promote tumor aggravation and poor survival in patients with osteosarcomas. Onco Targets Ther 2014, 7:477-484

35. Popper H: Mechanism of cell and tissue damage produced by immune reactions. Edited by Miescher GP. In Second International Symposium on Immunopathology. New York, NY: Grune and Stratton, 1962. pp. 303

36. Czaja AJ, Carpenter HA: Histologic findings in chronic hepatitis $\mathrm{C}$ with autoimmune features. Hepatology 1997, 26:459-466

37. Si L, Whiteside TL, VanThiel DH, Rabin BS: Lymphocytic subpopulations at the site of "piecemeal" necrosis in the end state chronic liver diseases and rejecting liver allografts in the cyclosporin-treated patients. Lab Invest 1984, 50:341

38. French SW, Nash S, Shitabata P, Kachi K, Hara C, Chedid A, Mendenhall CL: Pathology of alcoholic liver disease. Semin Liver Dis 1993, 13:154-169

39. Alexander G, Williams R: Characterization of the mononuclear cell infiltrate in piecemeal necrosis. Lab Invest 1984, 50:247

40. Huang JF, Yang Y, Sepulveda H, Shi W, Hwang I, Peterson PA, Jackson MR, Sprent J, Cai Z: TCR-mediated internalization of peptide-MHC complexes acquired by T cells. Science 1999, 286:952

41. Hwang I, Sprent J: Role of the actin cytoskeleton in T cell absorption and internalization of ligands from APC. J Immunol 2001, 166: $5099-5107$

42. Martinez-Martin N, Fernandez-Areans E, Cemerski S, Delgado P, Turner M, Heuser J, Irvine DJ, Huang B, Bustelo XR, Shaw A, Alarcon B: T cell receptor internalization from the immunological synapse is mediated by TC21 and RhoG GTPase-dependent phagocytosis. Immunity 2011, 35:208-222

43. French SW, Lu JG: Piecemeal necrosis is due to the immunologic synapse formation and internalization of intact TCR-MHCII complexes by CD4 T cells. Exp Mol Pathol 2018, 105:150-152

44. Takeuchi A, Saito T: CD4 CTL, a cytotoxic subset of CD4+ T cells, their differentiation and function. Front Immunol 2017, 8:194

45. Aslan N, Yurdaydin C, Wiegand J, Greten T, Ciner A, Meyer MF, Heiken H, Kuhlmann B, Kaiser T, Bozkaya H, Tillman HL, Bozdayi AM, Manns MP, Wedemeyer H: Cytotoxic CD4 T cells in viral hepatitis. J Viral Hepat 2006, 13:505-514

46. Appay V, Zaunders JJ, Papagno L, Sutton J, Jaramillo A, Waters A, Easterbrook P, Grey P, Smith D, McMichael AJ, Cooper DA, Rowland-Jones SL, Kelleher AD: Characterization of CD4(+) CTLs ex vivo. J Immunol 2002, 168:5954-5958

47. Juno JA, van Bockel D, Kent SJ, Kelleher AD, Zaunders JJ, Munier CM: Cytotoxic CD4 T cells: friend or foe during viral infection? Front Immunol 2017, 8:19

48. Geller SA, Petrovic LM: Biopsy Interpretation of the Liver. Philadelphia, PA, Lippincott Williams \& Wilkins, 2009. pp. 163-170

49. Lohr H, Manns M, Kyriatsoulis A, Lohse AW, Trautwein C, Meyer zum Buschenfelde KH, Fleischer B: Clonal analysis of liver-infiltrating T cells in patients with LKLM-1 antibody-positive autoimmune chronic active hepatitis. Clin Exp Immunol 1991, 84:297-302

50. Senaldi G, Portmann B, Mowat AP, Wieli-Vergani G, Vergani D: Immunohistochemical features of the portal tract mononuclear cell infiltrate in chronic aggressive hepatitis. Arch Dis Child 1992, 67: $1447-1453$

51. Oikawa T, Takahashi H, Ishikawa T, Hokari A, Otsuki N, Azuma M, Zeniya M, Tajiri H: Intrahepatic expression of the co-stimulatory molecules programmed death-1, and its ligands in autoimmune liver disease. Pathol Int 2007, 57:485-492

52. Wei CH, Penunun A, Karpouzas G, Fleishman W, Datta A, French SW: Troxis necrosis, a novel mechanism for drug-induced hepatitis secondary to immunomodulatory therapy. Exp Mol Pathol 2016, 99:341-343

53. Enbom TE, Le MD, Oesterich L, Rutgers J, French SW: Mechanism of hepatotoxicity due to black cohosh and electron microscopy analysis of two liver biopsies with clinical correlation. Exp Mol Pathol 2014, 96:279-283

54. Chen GC, Ramanthan YS, Law D, Funchain P, Chen GC, French S, Shlopov B, Eysselein V, Chung D, Reicher S, Pham BV: Acute liver injury induced by weight-loss herbal supplements. World J Hepatol 2010, 12:410-415

55. Rao R: Endotoxemia and gut barrier dysfunction in alcoholic liver disease. Hepatology 2009, 50:638-644

56. Mavrelis PG, Ammon HV, Gleysteen JJ, Komorowski RA, Charaf UK: Hepatic free fatty acids in alcoholic liver disease and morbid obesity. Hepatology 1983, 3:226-231

57. Lakshminarayanan V, Beno DW, Costa RH, Roebuck KA: Differential regulation of interleukin-8 and intercellular adhesion molecule1 by $\mathrm{H} 2 \mathrm{O} 2$ and tumor necrosis factor-alpha in endothelial and epithelial cells. J Biol Chem 1997, 272:32910-32918

58. Lytton SD, Helander A, Zhang-Gouillon ZZ, Stokkeland K, Bordone R, Arico S, Albano E, French SW, Ingelman-Sundberg M: Autoantibodies against cytochromes P-4502E1 and P-4503A in alcoholics. Mol Pharmacol 1999, 55:223-233

59. Albano E, French SW, Ingelman-Sundberg M: Cytochrome P4502E1, hydroxyethyl free radicals, and immune reactions associate with alcoholic liver disease. Alcohol Clin Exp Res 1998, 22: $739-742$

60. Albano E, French SW, Ingelman-Sundberg M: Hydroxyethyl radicals in ethanol hepatotoxicity. Front Bio Sci 1999, 4:dA533-dA540

61. Worrall S, De Jersey J, Shanley BC, Wilce PA: Ethanol induces the production of antibodies to acetaldehyde-modified epitopes in rats. Alcohol 1989, 24:217-223

62. Wang HJ, Gao B, Zakhari S, Nagy LE: Inflammation in alcoholic liver disease. Annu Rev Nutr 2012, 32:343-368

63. Spengler U, Pape GR, Hoffman RM, Johnson JP, Eisenburg J, Paumgartner G, Riethmuller G: Differential expression of MHC class II subregion products on bile duct epithelial cells and hepatocytes in patients with primary biliary cirrhosis. Hepatology 1988, 8:459-462

64. Van der Ord JJ, Sciot R, Desmet VJ: Expression of MHC products by normal and abnormal bile duct epithelium. J Hepatol 1986, 3: 310-317

65. Webb GJ, Siminovitch KA, Hirschfield GM: The immunogenetics of primary biliary cirrhosis: a comprehensive review. J Autoimmun 2015, 64:42-52

66. Xie XW, Mei MH, Liao WJ, Qian LH, Yu X, Fei R, Qin LL, Zhang HH, Peng JR, Shen DH, Wei L, Chen S: Expression of CIITArelated MHCII molecules in tumors linked to prognosis in hepatocellular carcinoma. Int J Oncol 2009, 34:681-688

67. Zhu AX, Finn RS, Edeline J, Cattan S, Ogasawara S, Palmer D, Verslype C, Zagonel V, Fartoux L, Vogel A, Sarker D, Verset G, Chan SL, Knox J, Daniele B, Webber AL, Ebbinghaus SW, Ma J, Siegal AB, Cheng AL, Kudo M; KEYNOTE-224 investigators: Pembrolizumab in patients with advanced hepatocellular carcinoma previously treated with sorafenib (KEYNOTE-224): a nonrandomised, open-label phase 2 trial. Lancet Oncol 2018, 19: 940-952

68. Finn SR, Baek-Yeol R, Philippe M, Kudo M, Bouattour M, Lim HY, Vladimirovich Breder V, Edeline J, Chao Y, Ogasawara S, Yau T, Garrido M, Chan SL, Knox JJ, Daniele B, Ebbinghaus S, Chen E, Siegel AB, Zhu AX, Cheng AL: Results of KEYNOTE-240: phase 3 study of pembrolizumab (Pembro) vs best supportive care (BSC) for second line therapy in advanced hepatocellular carcinoma (HCC). J Clin Oncol 2019, 37:4004

69. Larkin J, Chiarion-Sileni V, Gonzalez R, Grob JJ, Cowey L, Lao CD, Schadendorf D, Dummer R, Smylie M, Rutkowski P, Ferrucci PF, Hill A, Wagstaff J, Carlino MS, Haanen JB, Maio M, MarquezRodas I, McArther GA, Ascierto PA, Long GV, Callahan MK, 
Postow MA, Grossman K, Sznol M, Dreno B, Bastholt L, Yang A, Rollin LM, Horak C, Hodi FS, Wolchok JD: Combined nivolumab and ipilimumab or monotherapy in untreated melanoma. N Engl J Med 2015, 373:23-34

70. Johnson DB, Beckermann KE, Wang DY: Immune checkpoint inhibitor therapy in patients with autoimmune disease. Oncology (Williston Park) 2018, 32:190-194
71. Kato Y, Otsuka A, Miyachi Y, Kabashima K: Exacerbation of psoriasis vulgaris during nivolumab for oral mucosal melanoma. J Eur Acad Dermatol Venereol 2016, 30:e89-e91

72. Chae YK, Chiec L, Mohindra N, Gentzler R, Patel J, Giles F: A case of pembrolizumab-induced type-1 diabetes mellitus and discussion of immune checkpoint inhibitor-induced type 1 diabetes. Cancer Immunol Immunother 2017, 66:25-32 